\title{
BMJ Open Burden of heart failure in Flemish general practices: a registry-based study in the Intego database
}

\author{
Miek Smeets, ${ }^{1}$ Bert Vaes, ${ }^{1,2}$ Pavlos Mamouris, ${ }^{1}$ Marjan Van Den Akker, ${ }^{1,3}$ \\ Gijs Van Pottelbergh, ${ }^{1}$ Geert Goderis, ${ }^{1}$ Stefan Janssens, ${ }^{4}$ Bert Aertgeerts, ${ }^{1}$ \\ Séverine Henrard ${ }^{1,2}$
}

\begin{abstract}
To cite: Smeets M, Vaes B, Mamouris $\mathrm{P}$, et al. Burden of heart failure in Flemish general practices: a registrybased study in the Intego database. BMJ Open 2019;9:e022972. doi:10.1136/ bmjopen-2018-022972

- Prepublication history and additional material for this paper are available online. To view these files, please visit the journal online (http://dx.doi. org/10.1136/bmjopen-2018022972).
\end{abstract}

Received 21 March 2018 Revised 27 August 2018 Accepted 17 October 2018

Check for updates

(C) Author(s) (or their employer(s)) 2019. Re-use permitted under CC BY-NC. No commercial re-use. See rights and permissions. Published by BMJ.

${ }^{1}$ Departement of Public Health and Primary Care, Katholieke Universiteit Leuven, Leuven, Belgium

${ }^{2}$ Louvain Drug Research Institute, Clinical Pharmacy Research Group and Institute of Health and Society (IRSS), Université catholique de Louvain (UCL), Brussels, Belgium ${ }^{3}$ Department of Family Medicine, Care and Public Health Research Institute, Maastricht University, Maastricht, Limburg, The Netherlands

${ }^{4}$ Departement of Cardiovascular Diseases, Universitair

Ziekenhuis Gasthuisberg, KU Leuven, Leuven, Belgium

Correspondence to

Dr Miek Smeets;

miek.smeets@kuleuven.be

\section{ABSTRACT}

Objectives To assess the prevalence and incidence of heart failure (HF) stages $\mathrm{A}$ to $\mathrm{C} / \mathrm{D}$ and their evolution over a 16-year period. Additionally, trends in comorbidities and cardiovascular (CV) treatment in patients with HF were studied in the same period.

Design Registry-based study.

Setting Primary care, Flanders, Belgium.

Participants Data were obtained from Intego, a morbidity registration network in which 111 general practitioners of 48 practices collaborate. In the study period between 2000 and 2015, data from 165796 unique patients aged 45 years and older were available.

Outcome measures Prevalence and incidence were calculated for HF stage $A, B$ and $C / D$ by gender. Additionally, the trend in age-standardised prevalence and incidence rates between 2000 and 2015 was analysed with joint-point regression. The same model was used to study trends in comorbidity profiles in incident HF cases and trends in cardiovascular medication in prevalent HF cases.

Results We found a downward trend in the incidence and prevalence of $\mathrm{HF}$ stage $\mathrm{C} / \mathrm{D}$ in Flemish general practice between 2000 and 2015, whereas the prevalence and incidence of stage $A$ and $B$ increased. The burden of comorbidities in incident HF cases increased during the study period, as shown by an increasing disease count $(p<0.001)$. The prescription of cardiovascular medication such as renin-angiotensin-aldosterone system blockade, $\beta$-blockers and statins showed a sharp increase in the first part of the study period (2000-2008).

Conclusion Age-standardised incidence and prevalence of $\mathrm{HF}$ stage $\mathrm{C} / \mathrm{D}$ showed a slightly downward trend over the past 16 years, probably due to the sharp increase in cardiovascular treatment. However, the increasing agestandardised incidence and prevalence of stage $A$ and $B$, as precursors of symptomatic $\mathrm{HF}$, together with a rising comorbid burden, highlights the challenges we are still facing.

\section{INTRODUCTION}

Healthcare and social security systems in industrialised countries are faced with ageing populations. As a consequence, the prevalence of multimorbid patients taking multiple medications is rising. ${ }^{1}$ In this context

\section{Strengths and limitations of this study}

- The Intego database provides real-world data of 165796 unique patients aged 45 years and older, representative of the general Flemish population.

- The Intego database lends itself perfectly for studying trends, with data for a 16-year study period (2001-2015)

- This is the first community study to describe trends in incidence and prevalence of all heart failure stages and the first to describe trends in comorbidities and cardiovascular medication from 2008 to 2015.

- Completeness of the data in the Intego database depends on the quality of registration of the participating general practitioners (GPS). To this end, only optimal registration practices are included in the Intego database.

- Some diagnoses or comorbidities could not be reliably extracted from the Intego database because GPs were not used to registering them in a coded manner.

of emerging multimorbidity, heart failure (HF) has been shown to be associated with the highest number of comorbid diseases in an elderly population. ${ }^{2}$ Multimorbidity is associated with poor quality of life, physical disability, intensive healthcare use, multiple medications and increased risk of adverse drug events and mortality. ${ }^{3-5}$ Moreover, multimorbidity and polypharmacy are frequently reported barriers by general practitioners (GPs) to adequate diagnosis and management of patients with HF. ${ }^{6}$

$\mathrm{HF}$ is a progressive disease starting from cardiovascular (CV) risk factors, to asymptomatic changes in cardiac function and structure and eventually symptomatic HF and death. ${ }^{78}$ The American Heart Association (AHA) has developed a classification system for $\mathrm{HF}$ with stages A to D. ${ }^{7}$ A high prevalence of HF stage A and B in community patients older than 45 years and progressively worsening 5-year survival rates for each stage were 
described in former studies. ${ }^{9}{ }^{10}$ Early detection of stages $\mathrm{A}$ and $\mathrm{B}$ and subsequent intervention can lead to longterm reduction in morbidity and mortality of HF. ${ }^{11}{ }^{12}$ Recently, a large population-based study confirmed the rising burden of $\mathrm{HF}$ stage $\mathrm{C} / \mathrm{D}$, highlighting the need of prevention ${ }^{13}$; however, general practice data about the number of patients at risk for $\mathrm{HF}$ and evolution of the $\mathrm{CV}$ burden over time are limited.

Therefore, this study assessed the prevalence and incidence of HF stages A to C/D in Flemish general practice and their evolution over a 16-year period. Additionally, trends in comorbidities and cardiovascular treatment in patients with $\mathrm{HF}$ in general practice were described over the same period.

\section{METHODS}

\section{Data collection}

Data were obtained from Intego, a Belgian general practice-based morbidity registration network at the Department of General Practice of the University of Leuven. ${ }^{14}$ In 2015, 111 general practitioners (GPs) of 48 practices evenly spread throughout Flanders, Belgium, collaborated in the Intego project. GPs applied for inclusion in the registry. Before acceptance of their data, registration performance was audited using algorithms to compare their results with those of all other applicants. Only data from practices with optimal registration performance were included in the database. Additionally, Intego data have been externally validated by means of national and international comparisons. Intego GPs prospectively and routinely registered all new diagnoses and new drug prescriptions, as well as laboratory test results and patient information, using computer-generated keywords internally linked to codes. Using specially framed extraction software, new data were encrypted and collected from the GPs' personal computers and entered in a central database on a yearly basis. Registered data were continuously updated and historically accumulated for each patient. New diagnoses were classified according to a very detailed thesaurus (Medidoc codes) automatically linked to the International Classification of Primary Care (ICPC-2) and International Statistical Classification of Diseases and Related Health Problems 10th Revision. Drugs were classified according to the WHO's Anatomical Therapeutic Chemical (ATC) classification system. ${ }^{15}$ The denominator was the yearly contact group (YCG). These are the patients that visited the practice at least once in a given year. ${ }^{1416}$ The present study used Intego data of a 16-year time period from 1 January 2000 to 31 December 2015.

\section{Patient involvement}

No patients were involved in the development of the research question, study design or interpretation of the data.

\section{Definition of heart failure stages}

HF stage 0 was defined as absence of HF risk factors in healthy subjects. ${ }^{7}$ HF stage A was defined as the presence of HF risk factors (cardiovascular risk factors) without a structural or functional cardiac abnormality. ${ }^{7}$ In accordance with previous studies, only risk factors that have been shown to be predictive of HF in longitudinal studies were used (hypertension, diabetes mellitus, obesity and atherosclerotic diseases with exclusion of myocardial infarction). ${ }^{9}$ However, obesity could not reliably be assessed from the registry. HF stage B was defined as structural or functional heart disease without HF symptoms including acute myocardial infarction, heart valve disease, cardiomyopathy or tachyarrhythmia. ${ }^{7}$ Patients with HF stage A or B were identified based on an ICPC-2 coded diagnosis in their Electronic Medical Record (EMR). An overview of all used codes can be found in online supplement 1. Patients with HF stage C/D were considered patients with an ICPC-2 coded diagnosis K77 in their EMR. The differentiation between stage C (symptomatic HF) and D (advanced HF, New York Heart Association IV) could not be made. The flow chart followed to extract the prevalence and incidence of HF stage A-C/D can be found in online supplement 2 .

\section{Comorbidities and cardiovascular treatment}

Medical history previous to the date of HF diagnosis was registered for all incident HF cases between 2000 and 2015. A disease count was calculated for all incident HF cases. For this disease count, a list of chronic diseases based on the paper by Knottnerus et al was used, with exclusion of heart failure (K77) (see online supplement 3) ${ }^{17}$ For the presence of chronic kidney disease, the glomerular filtration rate was estimated (Modification of Diet in Renal Disease equation (MDRD) equation) based on the closest creatinine measurement in the 2 years before or after the date of HF diagnosis.

CV medication (antihypertensive agents, lipid-modifying agents and anticoagulants/antiplatelet agents) was recorded for all prevalent HF cases each year between 2000 and 2015. Medication use in a specific year was considered positive when at least one prescription had been made in that year (see online supplement 4: used ATC codes).

\section{Data analysis}

Prevalence (/100 patients) and incidence (/1000 patientyears at risk) were calculated for stage $\mathrm{A}, \mathrm{B}$ and $\mathrm{C} / \mathrm{D}$ by gender. The rates were age-standardised by taking the Flemish population in Belgium as the standard population. Ten-year age groups were formed starting from 45 to 55 with 85 years and older as the last group for standardisation. The reference year for the standard population was 2000. Additionally, the trend in age-standardised rates between 2000 and 2015 was analysed. For that purpose, a joinpoint regression analysis was performed. ${ }^{18}$ A joinpoint is a point in the trend curve where a statistically significant change in trend over time is observed. A 
minimum number of three observations from a joinpoint to either end of the data, and a minimum number of four observations between two joinpoints were required. From the joinpoint regression model, the annual percentage change (APC) and the average annual percentage change (AAPC) were extracted. APC is calculated for each significant trend from a piecewise log-linear model on the logarithm of the age-standardised rate versus the year. AAPC represents the average of APC estimates per significant trend weighted by the corresponding trend length (number of years in the trend). The trend analysis using the joinpoint regression model was performed using the SEER*Stat software (Joinpoint Trend Analysis software from the Surveillance Research Programme of the US National Cancer Institute (available at http:// surveillance.cancer.gov/joinpoint)). Trends in comorbidity profile were explored in incident HF cases with the Cochran-Armitage test and the Jonckheere-Terpstra test over four time intervals of 4 years (2000-2003, 2004-2007, 2008-2011 and 2012-2015). The Cochran-Armitage test for trend is used when we try to associate our binary response with an ordinal variable with $\mathrm{k}$ categories. The
Jonckheere-Terpstra trend test is used to try and associate a continuous response with an ordinal variable with $\mathrm{k}$ categories. $^{19}$

Trends in CV medication over the years 2000-2015 were analysed in prevalent $\mathrm{HF}$ cases using a joinpoint regression analysis, as described above. A two-sided $p$ value $<0.05$ was considered to be statistically significant. These analyses were performed using R Software V.3.3.2 (Free Software Foundation, Boston, Massachusetts, USA) (DescTools and clinfun packages).

\section{RESULTS}

Trends in age-standardised prevalence and incidence of HF stages (2000-2015)

The total study population between 1 January 2000 and 31 December 2015 consisted of 165796 unique patients aged 45 years and older. The age-standardised prevalence of stage A increased from $27 \%$ in 2000 to $35 \%$ in 2015 with a similar baseline prevalence and trend in men and women (AAPC 1.6, 95\% CI 1.3 to 1.9) (table 1). The same rising trend was observed in the age-standardised

Table 1 Trends in age-standardised prevalence and incidence of all HF stages (2000-2015)

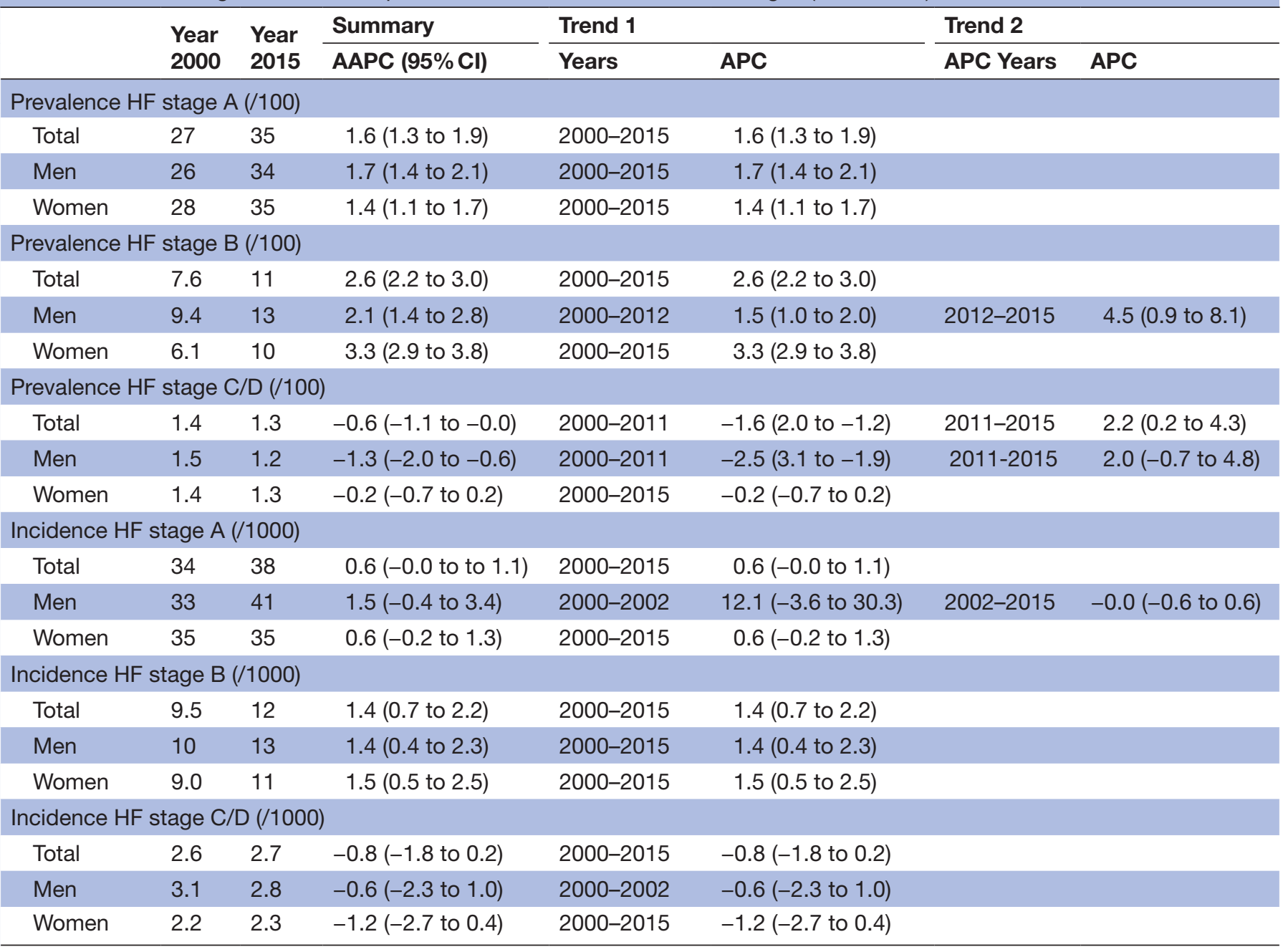

AAPC, average annual percentage change; APC, annual percentage change; HF, heart failure. 

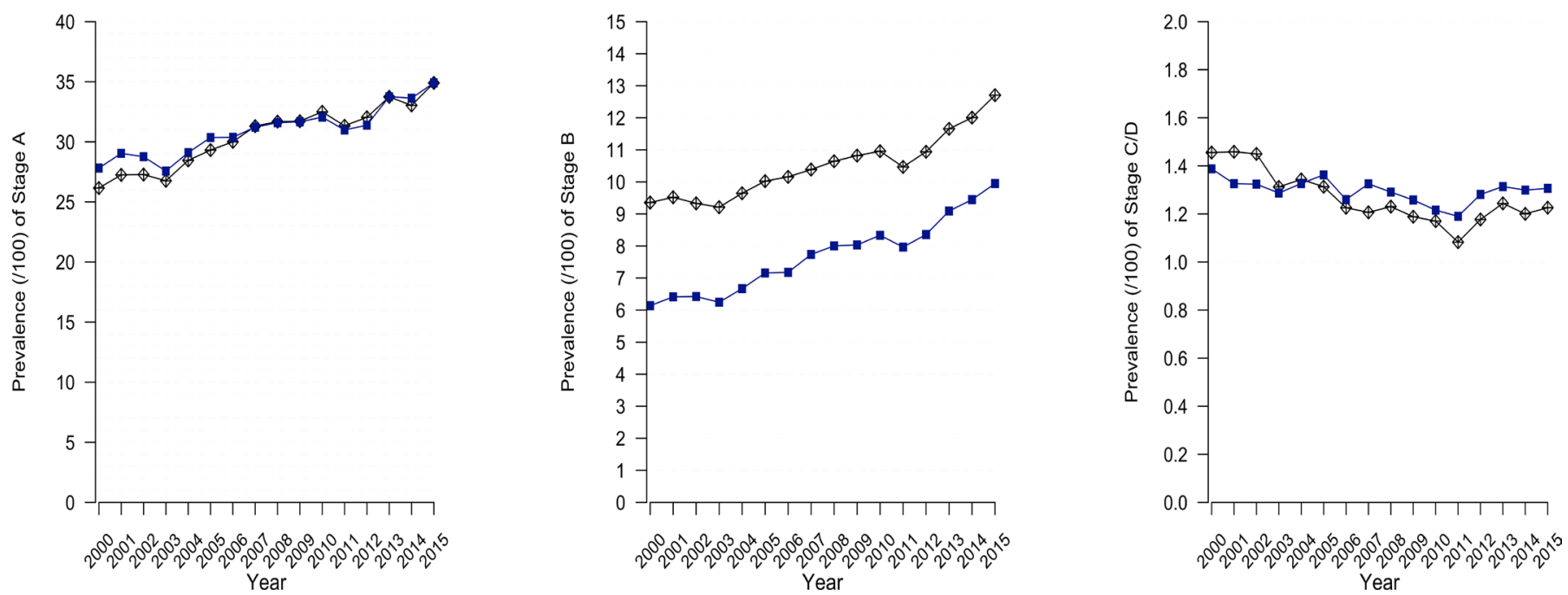

Figure 1 Age-standardised prevalence of different heart failure stages 2000-2015.

prevalence of stage B (from $7.6 \%$ in 2000 to $11 \%$ in 2015). A significant difference between the trend in men and women was noted. The prevalence of stage B showed a steeper rise for women than for men (AAPC difference men-women $-1.2,95 \%$ CI -2.0 to -0.4 ) but remained lower in women compared with men in 2015 $(10 \%$ vs $14 \%)$. For stage C/D, a general downward trend between 2000 and 2015 was noted (AAPC -0.6, 95\% CI -1.1 to 0.0 ) ( $1.54 \%$ vs $1.53 \%$ ) (figure 1 and table 1 ). In general, most trends in prevalence and incidence of the HF stages accounted for the whole study period and were reflected best by reporting AAPC.

The incidence of stage A (34/1000 in 2000 to 38/1000 in 2015) and stage B (10/1000 in 2000 to $13 / 1000$ in 2015) showed a slightly positive trend (AAPC $0.6,95 \%$ CI 0.0 to 1.1 and $1.4,95 \% \mathrm{CI} 0.7$ to 2.2 , respectively) while the incidence of stage C/D (2.6/1000 to 2.7/1000) showed a non-significant downward trend (AAPC -0.8 , $95 \%$ CI -1.8 to 0.2 ) (table 1 ).
The prevalence of stage $\mathrm{C} / \mathrm{D}$ increased with age (figure 2) with a prevalence of $0.12 \%$ in patients aged $45-54$ years, rising to $0.47 \%$ in patients aged $55-64$ years, $1.40 \%$ in patients aged $65-74$ years going to $3.5 \%$ in patients aged 75-84 years and $8.0 \%$ in patients aged 85 years and older in 2015 (see online supplement 2). Stage $\mathrm{A}$ and $\mathrm{B}$ prevalence also showed an increase with age. However, while stage $\mathrm{C} / \mathrm{D}$ is unlikely at a younger age, stage A and B already affected younger patients (figure 2).

\section{Trends in comorbidities in patients with HF stage C/D (2000- 2015)}

Among patients with HF stage $\mathrm{C} / \mathrm{D}$, the mean age of HF diagnosis increased significantly in the 2000-2015 period (77.3-78.3 years, $\mathrm{p}=0.004$ ), while a non-significant decline was noted in the proportion of women $(52 \%-$ $48 \%, \mathrm{p}=0.20$ ) (table 2). Additionally, the mean disease count of patients with HF showed a significant increase in the study period ranging from 3.3 to $4.3(\mathrm{p}<0.001)$,
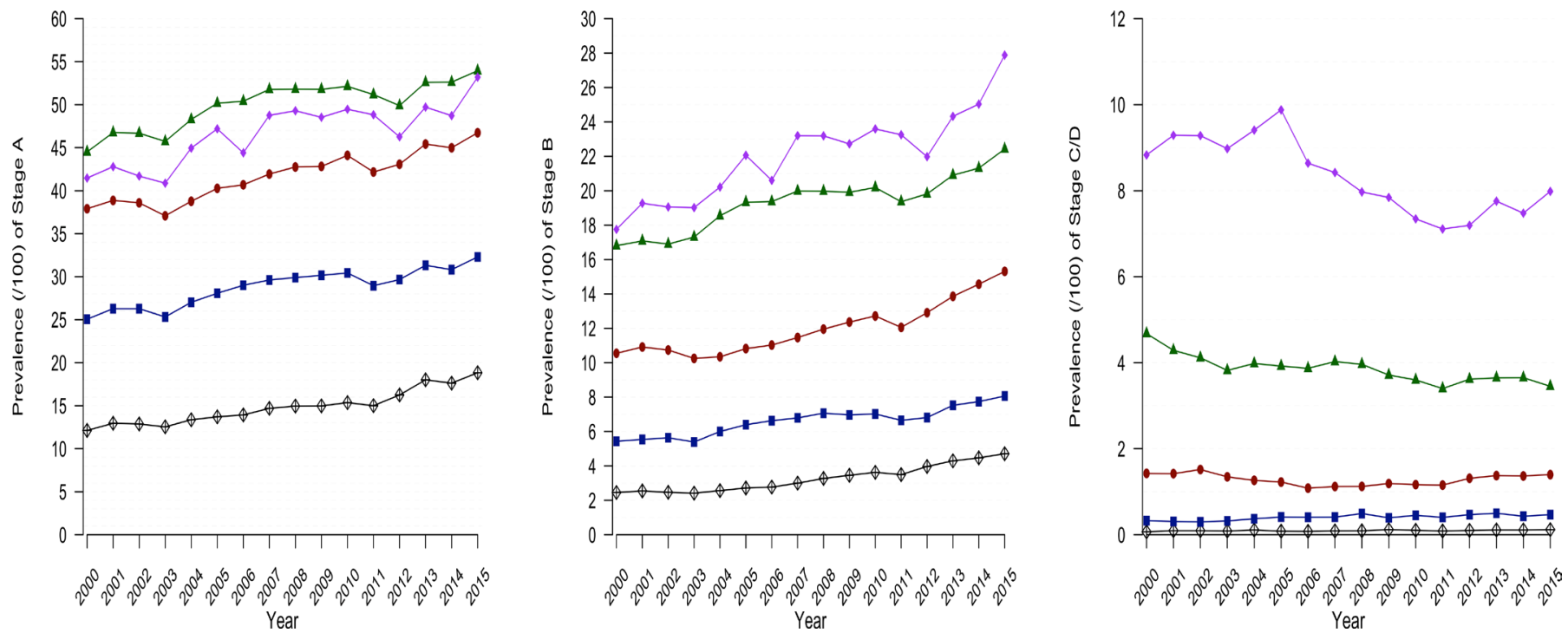

Figure 2 Prevalence of different heart failure stages 2000-2015 per age group. 
Table 2 Trends in comorbidities in patients with HF stage C/D (2000-2015)

\begin{tabular}{|c|c|c|c|c|c|}
\hline & 2000-2003 & 2004-2007 & 2008-2011 & 2012-2015 & $\begin{array}{l}\text { Trend test } \\
\mathrm{P} \text { value }\end{array}$ \\
\hline \multicolumn{6}{|l|}{ Study population } \\
\hline Patients with HF stage C/D & $n=515$ & $\mathrm{n}=627$ & $n=690$ & $n=648$ & \\
\hline Mean age $\pm S D$, years & $77.3 \pm 10.5$ & $78 \pm 10.2$ & $78.4 \pm 10.8$ & $78.3 \pm 10.9$ & 0.004 \\
\hline Women, n (\%) & $268(52)$ & $335(53)$ & $377(55)$ & $311(48)$ & 0.20 \\
\hline \multicolumn{6}{|l|}{ Prevalence of comorbidities, $n(\%)$} \\
\hline \multicolumn{6}{|l|}{ Cardiovascular comorbidities } \\
\hline Ischaemic heart disease (K74-K75-K76) & $160(31)$ & $198(32)$ & $206(30)$ & $204(31)$ & 0.95 \\
\hline Atrial fibrillation/flutter (K78) & $127(25)$ & $168(27)$ & $185(27)$ & $189(29)$ & 0.10 \\
\hline Heart valve disease (K83) & $54(11)$ & $91(15)$ & $111(16)$ & $110(17)$ & 0.002 \\
\hline Hypertension (K86-K87) & $182(35)$ & $263(42)$ & $319(46)$ & $310(48)$ & $<0.001$ \\
\hline Cerebrovascular disease (K90-K91) & $59(11)$ & $74(11)$ & $84(11)$ & $74(10)$ & 0.98 \\
\hline Diabetes mellitus (T89-T90) & $113(21)$ & $139(22)$ & $184(26)$ & $169(26)$ & 0.031 \\
\hline Lipid disorder (T93) & $85(17)$ & $137(22)$ & $168(24)$ & $156(24)$ & 0.002 \\
\hline \multicolumn{6}{|l|}{ Other comorbidities } \\
\hline COPD (R95) & $59(11)$ & $107(17)$ & $117(17)$ & $111(17)$ & 0.019 \\
\hline Asthma (R96) & $32(6)$ & $67(11)$ & $77(11)$ & $80(12)$ & 0.001 \\
\hline Hyperthyroidism (T85) & $17(3)$ & $19(3)$ & $38(6)$ & $35(5)$ & 0.017 \\
\hline Hypothyroidism (T86) & $19(4)$ & $32(5)$ & $40(6)$ & $32(5)$ & 0.31 \\
\hline Chronic kidney disease ${ }^{*}$ & $108(21)$ & $138(22)$ & $152(22)$ & $144(22)$ & 0.638 \\
\hline Depression (P76) & $58(11)$ & $90(14)$ & $94(14)$ & $91(14)$ & 0.27 \\
\hline Anxiety disorder (P74) & $7(7.3)$ & $12(7.7)$ & $16(9.2)$ & $22(12)$ & 0.018 \\
\hline Osteoarthritis (L89-90-91) & $121(23)$ & $185(29)$ & $209(30)$ & $212(32)$ & 0.001 \\
\hline Disease count of chronic diseases $†$ & 3.28 & 3.89 & 4.19 & 4.28 & $<0.001$ \\
\hline
\end{tabular}

*The glomerular filtration rate was estimated (MDRD equation) based on the closest creatinine measurement in the 2 years before or after the date of HF diagnosis.

†Full list of diseases: online supplement 3.

COPD, chronic obstructive pulmonary disease; HF, heart failure.

meaning that the comorbid burden of patients with HF increased. This was reflected in the trends of individual cardiovascular and non-cardiovascular comorbidities. The proportion of patients with cerebrovascular disease, hypothyroidism, ischaemic heart disease and chronic kidney disease remained stable while the prevalence of depression increased non-significantly $(11 \%-14 \%, \mathrm{p}=0.27)$. All the other cardiovascular and non-cardiovascular comorbidities showed a significant increase between 2000 and 2015 with hypertension ending up as the leading cardiovascular comorbidity in patients with $\mathrm{HF}$, with a prevalence of $48 \%$, followed by ischaemic heart disease $(31 \%)$ and atrial fibrillation (29\%) (table 2).

Trends in cardiovascular medication in patients with HF stage C/D (2000-2015)

The prescription of diuretics, renin-angiotensin-aldosterone system (RAAS) blockade, $\beta$-blockers and statins all showed a rising trend in patients with HF stage C/D between 2000 and 2015 (table 3). However, for cardiovascular medication different trends were observed within the study period (APC). For RAAS blockade and $\beta$-blockers the increase in prescriptions was especially marked between 2000 and 2007-2008 (APC 5.7, 95\% CI 3.6 to 7.9 and 13, 95\% CI 9.1 to 17 , respectively) while between $2007-2008$ and 2015 prescriptions remained stable (APC $-1.8,95 \%$ CI -3.8 to 0.3 and $1.1,95 \%$ CI -0.8 to 3.1 , respectively). Statin prescriptions followed the same pattern with a sharp increase between 2000 and 2006 (APC 29, 95\% CI 19 to 39), and kept on rising between 2006 and 2015, although at a weaker extent (APC 5.4, 95\% CI 3.2 to 7.7). This resulted in a prescription rate of $36 \%$ for RAAS blockade, $42 \%$ for $\beta$-blockers and 32\% for statins in 2015 (table 3).

Prescription of aldosterone antagonists (18\% in 2015) and calcium channel blockers (17\% in 2015) remained stable (AAPC $-0.9,95 \%$ CI -2.1 to 0.3 and $2.0,95 \%$ CI - 1.2 to 5.3, respectively), while prescription of cardiac glycosides clearly diminished (from $13 \%$ to $3.9 \%$ ), especially in later years (APC 2008-2015-14, 95\% CI -18 to -10).

\section{DISCUSSION}

This large registry-based study showed a downward trend in age-standardised incidence and prevalence of $\mathrm{HF}$ 


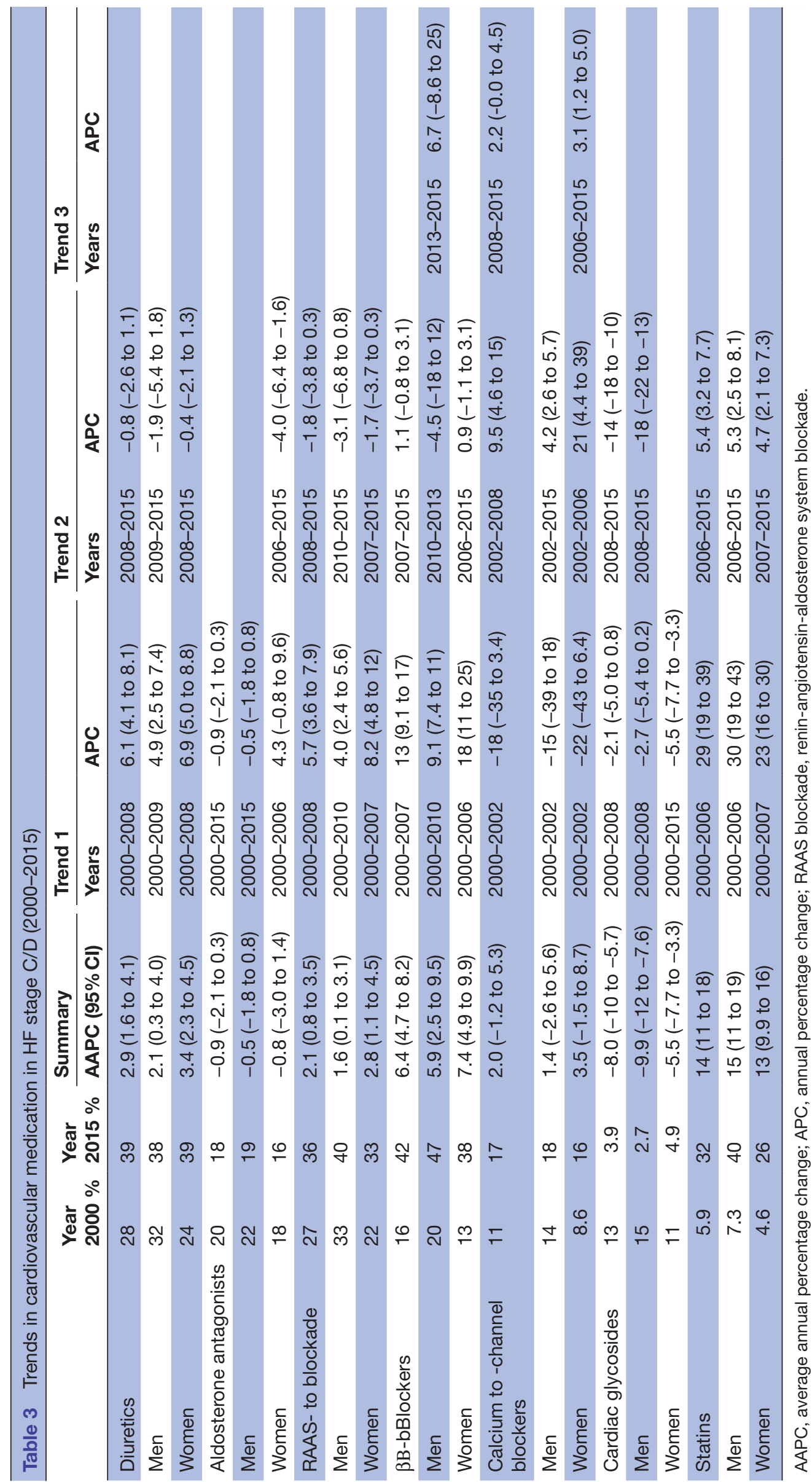


stage C/D in Flemish general practice between 2000 and 2015, while the prevalence and incidence of stage A and $\mathrm{B}$ did increase. The burden of comorbidities in incident HF cases increased during the study period, as shown by an increasing disease count. Although the prescription of cardiovascular medication such as RAAS blockade, $\beta$-blockers and statins showed a sharp increase in the first part of the study period (2000-2008), the prescription rates for 2015 remained lower than expected.

\section{Trends in age-standardised prevalence and incidence of HF stages \\ Downward trend in $\mathrm{HF}$ incidence and prevalence between 2000 and 2015}

We found a non-significant downward trend in age-standardised HF stage C/D incidence between 2000 and 2015. In previous research, three different trends in HF incidence were described. First, studies showed a decrease in the incidence of index HF hospitalisations in the last two decades. ${ }^{20-24}$ Second, a shift from incident index HF hospitalisations to incident community $\mathrm{HF}$ diagnoses (less severe HF) was noted. ${ }^{22}$ Third, even in the community, a decrease in age-standardised incident and prevalent HF was reported, explained by an increase in cardiovascular treatment. ${ }^{1324-26}$ The latter was confirmed in our findings and is a plausible explanation for the downward trend we found for HF incidence and prevalence in the Intego database. Another explanation might be that advances in cardiovascular treatment have led to less obvious presentation of incident HF cases and an increase in missed diagnoses or missed registration of $\mathrm{HF}$ cases over time. ${ }^{22}{ }^{25}$ Additionally, crude stage C/D prevalence rates and the total number of HF hospitalisations is still rising since ageing fuels the HF epidemic. ${ }^{13202226}$ The latter, together with the increasing prevalence and incidence of HF stage A and B and small downward trend in incidence and prevalence of HF in Flemish general practice demonstrates the ongoing importance of prevention of HF and early treatment of all HF stages. ${ }^{711} 13$

\section{Underestimation of stage $\mathrm{B}$ and $\mathrm{C}$ prevalence}

Crude prevalence rates depend highly on the method used to identify patients with $\mathrm{HF}^{27} 28$ Screening a community population led to prevalence rates for all $\mathrm{HF}$ stages ranging from $22 \%^{9}$ to $37 \%{ }^{10}$ for stage A, $34 \%^{9}$ to $43 \%{ }^{10}$ for stage B and $12 \%{ }^{9}$ to $9 \%{ }^{10}$ for HF stage C. Our observed prevalence of $35 \%$ for stage A in 2015 is in line with these results. However, for stage B (11\% in 2015) and C/D (1.3\% in 2015) a large discrepancy was noted, probably explained by the active use of echocardiography as the screening method in these former studies. ${ }^{9}{ }^{10}$ Consequently, only a fraction of stage $\mathrm{B}$ and $\mathrm{C} / \mathrm{D}$ patients are recognised or registered as such in general practice. This is not a local problem since a prevalence of $1.3 \%$ of symptomatic HF perfectly matches other registry-based general practice studies. ${ }^{13} 27$ 29-33 However, this finding has important consequences. Inaccurate documentation of HF in EMR decreased the likelihood that beneficial medication was prescribed for patients with $\mathrm{HF}^{34}$ Additionally, with the emerging role of e-health, where many applications such as computerised decision support systems are triggered by a coded diagnosis, registration of these diagnoses is crucial. Since identification of patients with $\mathrm{HF}$ is a prerequisite for adequate and timely treatment, further research is needed on strategies to improve detection and registration of HF in general practice.

\section{Trends in comorbidities in patients with HF stage C/D (2000- 2015)}

Increasing comorbid burden in patients with $\mathrm{HF}$

HF almost never occurs as an isolated condition. ${ }^{35-37}$ A former community study found that the proportion of patients with self-reported HF who had five or more comorbid conditions increased from $42.1 \%$ to $58 \%$ between 1988 and $2008 .^{38}$ Our study, in line with a recent study of Tran et al, ${ }^{39}$ confirms that this rising trend is still continuing. This is probably closely related to the increasing age of patients with, as shown in our study and others. ${ }^{35} 38-40$ Additionally, a rising trend in diagnoses, registration of diagnoses and survival may account for the increase in multimorbidity, together with (negative) lifestyle changes with respect to smoking, physical inactivity and diet. ${ }^{391}$ Furthermore, our data confirm the phenotype of general practice patients with HF as older female patients with hypertension rather than ischaemic heart disease. ${ }^{28}$ These findings have two important implications. First, a high and still rising comorbid burden complicates management of patients with $\mathrm{HF}$ and is a major contributor of (re)hospitalisations and mortality. ${ }^{36} 4243$ Recommendations for HF management should take this into account. If this finding is not included, recommendations for HF management only apply to a minority of the community patients with HF. ${ }^{394} 45$ Second, the same applies to randomised controlled trials excluding patients with concomitant chronic conditions targeting younger, male patients with ischaemic heart disease and higher drug prescription rates at baseline, thus not reflecting the community reality. ${ }^{28} 46$

\section{Trends in cardiovascular medication in patients with HF stage C/D (2000-2015)}

Increasing prescription rates of HF medication

Trends in prescription rates have been described, but only until $2008^{38}$ and not in a community setting. ${ }^{47}$ Hirt et al depicted a steady rising trend of RAAS blockade and $\beta$-blockers by comparing their data collected in $2015^{48}$ with those of the IMPROVEMENT-HF study collected in $2000{ }^{49}$ However, ours is the first general practice study to show that prescription rates increased sharply until 2007-2008 and remained stable afterwards. Since most of the major changes in pharmacological recommendations occurred around the millennium, a sharp increase was to be expected in the first years after 2000. However, does the stabilisation from 2008 onwards imply that there is no room left for improvement? Two recent German studies confirmed that prescription rates of RAAS blockade 
$(87 \%)^{48}$ and $\beta$-blockers $(84 \%)^{48}$ are currently high in general practice, ${ }^{48}$ although reaching target doses of these medications could still be substantially improved. ${ }^{48}$ These high prescription rates were not reflected in our study, with only $36 \%$ of patients with HF treated with RAAS blockade in 2015 and $42 \%$ with $\beta$-blockers. This could be explained by the lack of differentiation between HF with reduced ejection fraction (HFrEF) and HF with preserved ejection fraction (HFpEF), since no evidencebased treatment options exist for the latter. Additionally, the prevalence of HFpEF keeps increasing, especially in the community, compared with a decreasing HFrEF prevalence. ${ }^{51}$ Furthermore, our data depend on the quality of registration. EMR registration of medication can be hampered in patients in long-term care facilities, in patients visited at home and in medication prescribed by specialists. $^{48}$

In general, in the period we studied, the use of medications influencing survival in HFrEF patients (RAAS blockade, $\beta$-blockers) has increased, whereas the use of medications that were demonstrated not to improve survival (cardiac glycosides) has decreased. ${ }^{38} 48$

\section{Strengths and limitations}

The major strengths of the current study are the inclusion of a large real-world study population, representative of the general Flemish population, and the long follow-up period. This is the first registry-based study to describe trends in prevalence and incidence of all HF stages and the first to describe trends in comorbidities and cardiovascular medication from 2008 until 2015.

However, this study also has a few limitations. Patients are not registered with a particular GP in Belgium. The current study used the denominator. Former research has shown that the YCG accounts for $80 \%$ of the total practice population. ${ }^{16}$ The YCG is not the perfect denominator but it is the most realistic approach in countries without capitation. ${ }^{16}$ Additionally, one can assume that most patients with HF will visit their GP at least once a year. Furthermore, by using data from the Intego registry we can only extract data registered by GPs in the EMR, with respect to both coded diagnoses and medication prescriptions. This implies that certain trends could be influenced by changes in coding practices. Additionally, crude prevalence rates or prescription rates are influenced by the quality of registration, as explained above. Furthermore, some comorbidities such as obesity (stage A diagnosis) could not be reliably assessed in the Intego registry. The same applies for the difference between HFrEF and HFpEF, which could not be extracted from the Intego database, hindering robust conclusions about the quality of HF treatment. Importantly, mortality data are lacking. Therefore, patients in the YCG were considered at risk until the diagnosis or until December 31 of that specific year to calculate the incidence.

\section{CONCLUSION}

This large registry-based study in Flemish general practice covering a 16-year period showed a downward trend in age-standardised HF stage C/D incidence and prevalence. With a clear increase in HF stage A and B, as precursors of symptomatic HF, the burden of HF in Flemish general practice remains real. Moreover, the comorbid burden of patients with HF continues to increase. On a more positive note, prescriptions of beneficial cardiovascular medication showed a sharp increase, especially between 2000 and 2007-2008. Challenges for the future are improving detection and registration of patients with HF stage $\mathrm{C} / \mathrm{D}$ and the management of this highly complex patient population.

Acknowledgements The authors would like to thank all the participating general practitioners.

Contributors SH and PM performed the analyses and MS, BV and PM wrote the manuscript. MVDA, GVP and GG are responsible for the study concept and design and the recruitment of subjects and acquisition of data. All authors participated in the interpretation of the data. All authors approved the final version of the manuscript.

Funding Intego is funded on a regular basis by the Flemish Government (Ministry of Health and Welfare). We hereby state the independence of the researchers from the funders.

Competing interests None declared.

Patient consent for publication Not required.

Ethics approval The Intego procedures were approved by the ethical review board of the KULeuven Faculty of Medicine (no. ML 1723) and by the Belgian Privacy Commission (no. SCSZG/13/079).

Provenance and peer review Not commissioned; externally peer reviewed.

Data sharing statement All authors had full access to all data (including statistical reports and tables) in the study and assume responsibility for the integrity of the data and the accuracy of the data analyses.

Open access This is an open access article distributed in accordance with the Creative Commons Attribution Non Commercial (CC BY-NC 4.0) license, which permits others to distribute, remix, adapt, build upon this work non-commercially, and license their derivative works on different terms, provided the original work is properly cited, appropriate credit is given, any changes made indicated, and the use is non-commercial. See: http://creativecommons.org/licenses/by-nc/4.0/.

\section{REFERENCES}

1. van den Akker M, Buntinx F, Metsemakers JF, et al. Multimorbidity in general practice: prevalence, incidence, and determinants of co-occurring chronic and recurrent diseases. J Clin Epidemiol 1998;51:367-75

2. Marengoni A, Rizzuto D, Wang HX, et al. Patterns of chronic multimorbidity in the elderly population. J Am Geriatr Soc 2009;57:225-30.

3. Field TS, Gurwitz JH, Harrold LR, et al. Risk factors for adverse drug events among older adults in the ambulatory setting. J Am Geriatr Soc 2004;52:1349-54.

4. Gijsen R, Hoeymans N, Schellevis FG, et al. Causes and consequences of comorbidity: a review. J Clin Epidemiol 2001;54:661-74.

5. Hoffman C, Rice D, Sung HY. Persons with chronic conditions. Their prevalence and costs. JAMA 1996;276:1473-9.

6. Smeets M, Van Roy S, Aertgeerts B, et al. Improving care for heart failure patients in primary care, GPs' perceptions: a qualitative evidence synthesis. BMJ Open 2016;6:e013459.

7. Yancy CW, Jessup M, Bozkurt B, et al. WRITING COMMITTEE MEMBERSAmerican College of Cardiology Foundation/American Heart Association Task Force on Practice Guidelines. 2013 ACCF/ AHA guideline for the management of heart failure: a report of the American College of Cardiology Foundation/American 
Heart Association Task Force on practice guidelines. Circulation 2013;128:e240-e327.

8. Dzau V, Braunwald E. Resolved and unresolved issues in the prevention and treatment of coronary artery disease: a workshop consensus statement. Am Heart J 1991;121(4 Pt 1):1244-63.

9. Ammar KA, Jacobsen SJ, Mahoney DW, et al. Prevalence and prognostic significance of heart failure stages: application of the American College of Cardiology/American Heart Association heart failure staging criteria in the community. Circulation 2007;115:1563-70.

10. Jorge AL, Rosa ML, Martins WA, et al. The prevalence of stages of heart failure in primary care: a population-based study. J Card Fail 2016;22:153-7.

11. Subzposh F, Gupta A, Hankins SR, et al. Management of ACCF/AHA Stage A and B patients. Cardiol Clin 2014;32:63-71.

12. Ledwidge $\mathrm{M}$, Gallagher J, Conlon $\mathrm{C}$, et al. Natriuretic peptide-based screening and collaborative care for heart failure: the STOP-HF randomized trial. JAMA 2013;310:66-74.

13. Conrad N, Judge A, Tran J, et al. Temporal trends and patterns in heart failure incidence: a population-based study of 4 million individuals. Lancet 2018;391:572-80.

14. Truyers C, Goderis G, Dewitte H, et al. The Intego database: background, methods and basic results of a Flemish general practice-based continuous morbidity registration project. BMC Med Inform Decis Mak 2014:14:48.

15. Vaes B, Beke E, Truyers C, et al. The correlation between blood pressure and kidney function decline in older people: a registrybased cohort study. BMJ Open 2015;5:e007571.

16. Bartholomeeusen S, Kim CY, Mertens R, et al. The denominator in general practice, a new approach from the Intego database. Fam Pract 2005;22:442-7.

17. Knottnerus JA, Metsemakers J, Höppener P, et al. Chronic illness in the community and the concept of 'social prevalence'. Fam Pract 1992;9:15-21.

18. Kim HJ, Fay MP, Feuer EJ, et al. Permutation tests for joinpoint regression with applications to cancer rates. Stat Med 2000;19:335-51.

19. Alan A. Categorical data analysis. 2nd ed: Wiley, 2002.

20. Teng TH, Finn J, Hobbs M, et al. Heart failure: incidence, case fatality, and hospitalization rates in Western Australia between 1990 and 2005. Circ Heart Fail 2010;3:236-43.

21. Jhund PS, Macintyre K, Simpson CR, et al. Long-term trends in first hospitalization for heart failure and subsequent survival between 1986 and 2003: a population study of 5.1 million people. Circulation 2009;119:515-23.

22. Ezekowitz JA, Kaul P, Bakal JA, et al. Trends in heart failure care: has the incident diagnosis of heart failure shifted from the hospital to the emergency department and outpatient clinics? Eur J Heart Fail 2011;13:142-7.

23. Chen J, Hsieh AF, Dharmarajan $\mathrm{K}$, et al. National trends in heart failure hospitalization after acute myocardial infarction for Medicare beneficiaries: 1998-2010. Circulation 2013;128:2577-84.

24. Robertson J, McElduff P, Pearson SA, et al. The health services burden of heart failure: an analysis using linked population health data-sets. BMC Health Serv Res 2012;12:103.

25. Devroey D, Van Casteren V. The incidence and first-year mortality of heart failure in Belgium: a 2-year nationwide prospective registration. Int J Clin Pract 2010:64:330-5.

26. Mosterd A, Hoes AW. Clinical epidemiology of heart failure. Heart 2007;93:1137-46.

27. Guha K, McDonagh T. Heart failure epidemiology: European perspective. Curr Cardiol Rev 2013;9:123-7.

28. Smeets M, Henrard S, Aertgeerts B, et al. Methods to identify heart failure patients in general practice and their impact on patient characteristics: A systematic review. Int J Cardiol 2018;257:199-206.

29. Nilsson G, Strender LE. Management of heart failure in primary health care. A retrospective study on electronic patient records in a registered population. Scand J Prim Health Care 2002;20:161-5.
30. Murphy NF, Simpson CR, McAlister FA, et al. National survey of the prevalence, incidence, primary care burden, and treatment of heart failure in Scotland. Heart 2004;90:1129-36.

31. Majeed A, Williams J, de Lusignan S, et al. Management of heart failure in primary care after implementation of the National Service Framework for Coronary Heart Disease: a cross-sectional study. Public Health 2005:119:105-11.

32. Hawkins NM, Jhund PS, Simpson CR, et al. Primary care burden and treatment of patients with heart failure and chronic obstructive pulmonary disease in Scotland. Eur J Heart Fail 2010;12:17-24.

33. Hawkins NM, Scholes S, Bajekal M, et al. Community care in England: reducing socioeconomic inequalities in heart failure. Circulation 2012;126:1050-7.

34. Hartung DM, Hunt J, Siemienczuk J, et al. Clinical implications of an accurate problem list on heart failure treatment. J Gen Intern Med 2005;20:143-7.

35. van der Wel MC, Jansen RW, Bakx JC, et al. Non-cardiovascular co-morbidity in elderly patients with heart failure outnumbers cardiovascular co-morbidity. Eur J Heart Fail 2007;9(6-7):709-15.

36. Braunstein JB, Anderson GF, Gerstenblith G, et al. Noncardiac comorbidity increases preventable hospitalizations and mortality among Medicare beneficiaries with chronic heart failure. J Am Coll Cardiol 2003;42:1226-33.

37. Baron-Franco B, McLean G, Mair FS, et al. Comorbidity and polypharmacy in chronic heart failure: a large cross-sectional study in primary care. Br J Gen Pract 2017;67:e314-e320.

38. Wong CY, Chaudhry SI, Desai MM, et al. Trends in comorbidity, disability, and polypharmacy in heart failure. Am J Med 2011;124:136-43.

39. Tran J, Norton R, Conrad N, et al. Patterns and temporal trends of comorbidity among adult patients with incident cardiovascular disease in the UK between 2000 and 2014: A population-based cohort study. PLoS Med 2018;15:e1002513.

40. Liu L. Changes in cardiovascular hospitalization and comorbidity of heart failure in the United States: findings from the National Hospital Discharge Surveys 1980-2006. Int J Cardiol 2011;149:39-45.

41. Uijen AA, van de Lisdonk EH. Multimorbidity in primary care: prevalence and trend over the last 20 years. Eur J Gen Pract 2008;14 Suppl 1-28-32.

42. Oudejans I, Mosterd A, Zuithoff NP, et al. Comorbidity drives mortality in newly diagnosed heart failure: a study among geriatric outpatients. J Card Fail 2012;18:47-52.

43. Ahluwalia SC, Gross CP, Chaudhry SI, et al. Impact of comorbidity on mortality among older persons with advanced heart failure. $J$ Gen Intern Med 2012;27:513-9.

44. Tinetti ME, Bogardus ST, Agostini JV. Potential pitfalls of diseasespecific guidelines for patients with multiple conditions. $N$ Engl $J$ Med 2004;351:2870-4.

45. De Maeseneer J, Roberts RG, Demarzo M, et al. Tackling NCDs: a different approach is needed. Lancet 2012;379:1860-1.

46. Buffel du Vaure C, Dechartres A, Battin C, et al. Exclusion of patients with concomitant chronic conditions in ongoing randomised controlled trials targeting $10 \mathrm{common}$ chronic conditions and registered at ClinicalTrials.gov: a systematic review of registration details. BMJ Open 2016;6:e012265.

47. Nakano A, Johnsen SP, Frederiksen BL, et al. Trends in quality of care among patients with incident heart failure in Denmark 20032010: a nationwide cohort study. BMC Health Serv Res 2013;13:391.

48. Hirt MN, Muttardi A, Helms TM, et al. General practitioners adherence to chronic heart failure guidelines regarding medication: the GP-HF study. Clin Res Cardiol 2016;105:441-50.

49. Cleland JG, Cohen-Solal A, Aguilar JC, et al. Management of heart failure in primary care (the IMPROVEMENT of Heart Failure Programme): an international survey. Lancet 2002;360:1631-9.

50. Tebbe $U$, Tschöpe C, Wirtz JH, et al. Registry in Germany focusing on level-specific and evidence-based decision finding in the treatment of heart failure: REFLECT-HF. Clin Res Cardiol 2014;103:665-73.

51. Owan TE, Hodge DO, Herges RM, et al. Trends in prevalence and outcome of heart failure with preserved ejection fraction. $N$ Engl $J$ Med 2006;355:251-9. 